\title{
Sporadic auroras near the geomagnetic equator: in the Philippines, on 27 October 1856
}

\author{
Hisashi Hayakawa ${ }^{1,2,3}$, José M. Vaquero ${ }^{4}$, and Yusuke Ebihara ${ }^{5,6}$ \\ ${ }^{1}$ Graduate School of Letters, Osaka University, Toyonaka, 5600043, Japan \\ ${ }^{2}$ Science and Technology Facilities Council, RAL Space, Rutherford Appleton Laboratory, \\ Harwell Campus, Didcot, OX11 0QX, UK \\ ${ }^{3}$ Japan Society for the Promotion of Science, 5-3-1 Kojimachi, Chiyoda-ku, Tokyo 102-0083, Japan \\ ${ }^{4}$ Departamento de Física, Universidad de Extremadura, 06800 Mérida, Spain \\ ${ }^{5}$ Research Institute for Sustainable Humanosphere, Kyoto University, Uji, 6100011, Japan \\ ${ }^{6}$ Unit of Synergetic Studies for Space, Kyoto University, Kyoto, 6068306, Japan
}

Correspondence: Hisashi Hayakawa (hayakawa@kwasan.kyoto-u.ac.jp)

Received: 30 May 2018 - Discussion started: 11 June 2018

Accepted: 18 August 2018 - Published: 29 August 2018

\begin{abstract}
While low-latitude auroral displays are normally considered to be a manifestation of magnetic storms of considerable size, Silverman (2003, JGR, 108, A4) reported numerous "sporadic auroras" which appear locally at relatively low magnetic latitude during times of just moderate magnetic activity. Here, a case study is presented of an aurora near the geomagnetic equator based on a report from the Philippine islands on 27 October 1856. An analysis of this report shows it to be consistent with the known cases of sporadic auroras, except for its appearance at considerably low magnetic latitude. The record also suggests that an extremely low-latitude aurora is not always accompanied by large magnetic storms. The description of its brief appearance leads to a possible physical explanation based on an ephemeral magnetospheric disturbance provoking this sporadic aurora.
\end{abstract}

\section{Introduction}

It is known that a low-latitude aurora is a manifestation of a magnetic storm caused by solar eruptions (e.g. Gonzalez et al., 1994; Yokoyama et al., 1998; Shiokawa et al., 2005; Willis et al., 2006; Odenwald, 2015). Since the beginning of systematic magnetic observations in the mid-19th century, magnetic records have been compared with auroral displays (e.g. Allen et al., 1989; Yokoyama et al., 1998; Silverman, 1995, 2006, 2008; Silverman and Cliver, 2001; Shiokawa et al., 1998, 2005; Vaquero et al., 2008). In August and September 1859, solar eruptions from large sunspots caused an intense magnetic storm reaching values as extreme as $1600 \mathrm{nT}$ in the horizontal geomagnetic field at Colaba (Tsurutani et al., 2003; Nevanlinna, 2006; Ribeiro et al., 2011), with major auroral displays seen worldwide down to magnetic latitudes (hereafter, MLATs) as low as $\sim 20^{\circ}$ (Kimball, 1960; Cliver and Svalgaard, 2004; Green and Boardsen, 2006; Farrona et al., 2011; Cliver and Dietrich, 2013; Hayakawa et al., 2016b; Lakhina and Tsurutani, 2016).

However, it is reported that auroral displays at low MLATs also occur during low or moderate geomagnetic disturbances. Silverman (2003) examined these auroral displays at relatively low MLAT during low or moderate geomagnetic disturbances in the Climatological Data of the United States during 1880 to 1940 , identifying 54 cases in the United States, and attesting to the reality of "sporadic aurorae", using the terminology of Botley (1963), who defined this phenomenon as a "single ray in a sky otherwise seemingly clear of auroral light, or isolated patches well to the equatorial side of a great display", citing Abbe (1895).

Willis et al. (2007) and Vaquero et al. (2007, 2013) surveyed this kind of localized low-latitude auroral display in China, Spain, and Mexico to identify reports during low or moderate geomagnetic activity. Silverman (2003) and Willis et al. (2007) drew attention to the question of the mechanism behind them, as to how the localized auroral display can be 
seen at a low latitude without there being any intense magnetic storms.

In this short contribution, we aim to describe a case of a "sporadic aurora" in the Philippine islands, close to the geomagnetic equator. It should be noted that auroras near the geomagnetic equator have yet to be studied, and knowledge of them will be an important key to scientific understanding of "sporadic auroras".

\section{Material and method}

Antonio Llanos (1806-1881), a Spanish priest with an interest in botany and meteorology (Vaquero et al., 2005), reports a curious account of an "Observation of an aurora borealis in Manila (Observación de una aurora boreal en Manila)" (Llanos, 1857). As is explicit in the title, Llanos considered this phenomenon an "aurora borealis" while being aware that the appearance of an aurora at such low latitudes is extremely rare. He associates the appearance of this aurora with exceptional (and unknown) circumstances of the atmosphere, and therefore wrote up this report so that physicists working on the origin of the phenomenon would have evidence of this unusual observation.

Based on this historical report by Llanos, we shall consider the nature of this phenomenon, compute the contemporary MLAT of the observation site, and compare the record with contemporary geomagnetic activity. Systematic magnetic observations started in the 1840 s, and the $a k$ index has been available from 1844 onwards, while the $a a$ index has been available from 1868 (Nevanlinna, 2004; Willis et al., 2007). We examine the values of the $a k$ index (Nevanlinna and Kataja, 1993; Nevanlinna, 2004) around the date of observation provided by Llanos.

\section{The aurora borealis on 27 October 1856}

Antonio Llanos reported the auroral display to a Spanish journal entitled Revista de los Progresos de las Ciencias Exactas, Físicas y Naturales (see Fig. 1). We summarize his report and review his observation. First, we shall extract Llanos's description of the observational report.

“At this moment [at 9 o'clock at night], observing the cloudscape of the atmosphere, I noticed that, on the NW side, with a short difference there was a faint but weak white light on that horizon, which at first I supposed was produced by some cause, such as from a fire. In that part, there is a range of mountains that form the provinces of Balanga and Zambales. The illuminated space would only rise about $4^{\circ}$ above the horizon, and the segment width would be about $25^{\circ}$. It seemed to be on the skirt or side of these mountains opposite the NW, and as if it were stopped there, prevented its passage by the said mountain ranges. At its base, the light was noticed to be more clear and perceptible, and some more resplendent points could be seen in its mass, noting also some movement of vertical undulation which it manifested, sometimes stronger and sometimes weaker, until finally it disappeared, leaving total darkness. When I began to notice it, I found it in the said state, and the time of duration in my view would be some 5 minutes. That illumination had scarcely disappeared, when on the opposite side of the first quadrant, that is, in the $\mathrm{NE}$, the same phenomenon was repeated with the same circumstances as the previous one, although with a greater extension, there being also another mountain range called Gapang, which runs in the same direction from $\mathrm{N}$ to $\mathrm{S}$, finding myself in the basin that these two ranges comprise; but on this occasion it lasted longer, or double the first, and it was 10 minutes, with the wind firmly on the same side or a little more to the $\mathrm{E}$, and with quite a lot of rain."

\section{The observational site and its magnetic latitude}

Antonio Llanos explicitly writes his observational site as being Manila, and its geographical latitude as at "latitude $15^{\circ} \mathrm{N}$, a little more or less". We estimate his observational site as the old city area of Manila $\left(14^{\circ} 35^{\prime} \mathrm{N}, 120^{\circ} 58^{\prime} \mathrm{E}\right)$. We computed the contemporary MLAT for this place in 1856 , based on the dipole component of the GUFM1 geomagnetic field model (Jackson et al., 2000). We obtained the value of $3.3^{\circ}$ MLAT. This value in 1900 is within $0.05^{\circ}$ of difference from that in 1900 as computed by the IGRF model (Thébault et al., 2015). Therefore, one can fairly consider this observation to have been made near the geomagnetic equator.

It is not common for auroral displays to be seen anywhere near the geomagnetic equator. In some extreme magnetic storms, it is known that auroral displays were visible down to some $18^{\circ}$ to $30^{\circ}$ MLAT, such as those in the major storms of $1989,1921,1909,1872,1870,1859,1770$, and 1730 (Kimball, 1960; Allen et al., 1989; Silverman, 1995, 2006, 2008; Silverman and Cliver, 2001; Vaquero et al., 2008; Hayakawa et al., 2017, 2018a, b; Ebihara et al., 2017; Willis et al., 1996), as partially reviewed by Cliver and Svalgaard (2004) and Cliver and Dietrich (2013). However, this value $\left(3.3^{\circ}\right.$ MLAT) is evidently closer to the geomagnetic equator, and is much lower than in the other events.

\section{Nature of this phenomenon}

It is worth consideration as to whether this record of an "aurora borealis" can be related to other phenomena. Antonio Llanos suspected this phenomenon at first to be "as from a fire", and ended by describing it as a "meteor that is so rare at low northern latitudes" following his conclusion that it was indeed an "aurora boreal". Nonetheless, it is possible to find 
223

mos están, pudieran disolverse mútuamente sin combinarse, y coexistir en el boro cristalizado, sin que se alterase la forma mo, al cual está tan inmediata. Sabido es (y en este hecho mo, al cual está tan inmediata. Sabido es ( $\mathrm{y}$ en este hecho se
funda el método de separar ambos metales por cristalizacion) que el plomo cristaliza sin arrastrar cantidades notables de que el plomo cristaliza sin arrastrar cantidades notables de plata: sepáranse como una sal

Tienen cabida estas observaciones en el aluminio, cuya xistencia en el boro en cantidades muy variables (desde 0 hasta 13 por 100) nunca indica una combinacion, porque la fórmula $\mathrm{Al}, \mathrm{Bo} 0^{7}$ exijiria ya más de 20 por 100 de aluminio. Este hecho nuevo podrá servir en nuestro concepto para determinar las condiciones de isomorfia de los cuerpos simples: $\mathrm{y}$ aun pudiera apoyar la opinion de que debiera ponerse el aluminio en la serie del carbono y del boro con igual razon y en el mismo sitio que el antimonio en la del ázoe y el fósforo. Y véase aqui una aplicacion del método paralélico, que tantos servicios lleva prestados á las ciencias naturales.

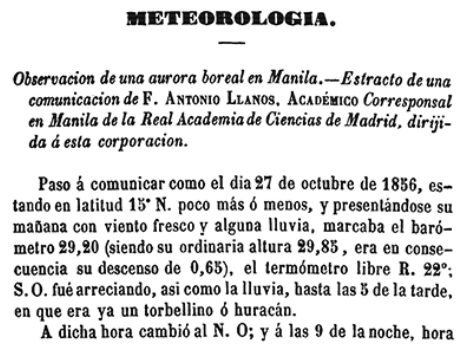

221

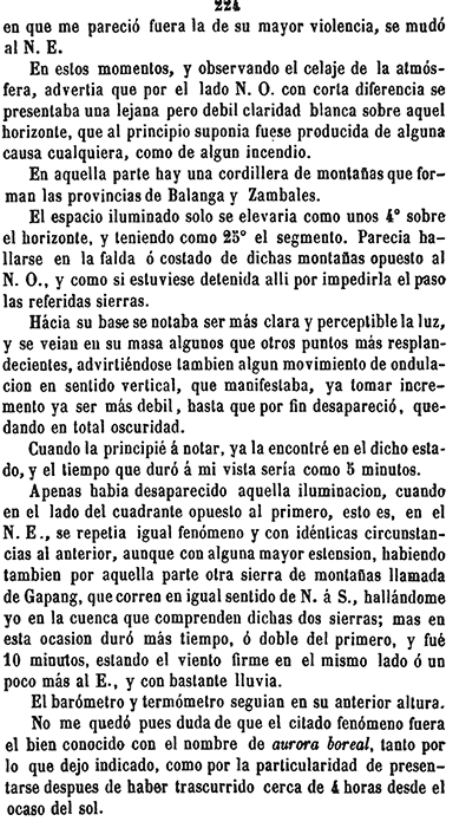

225

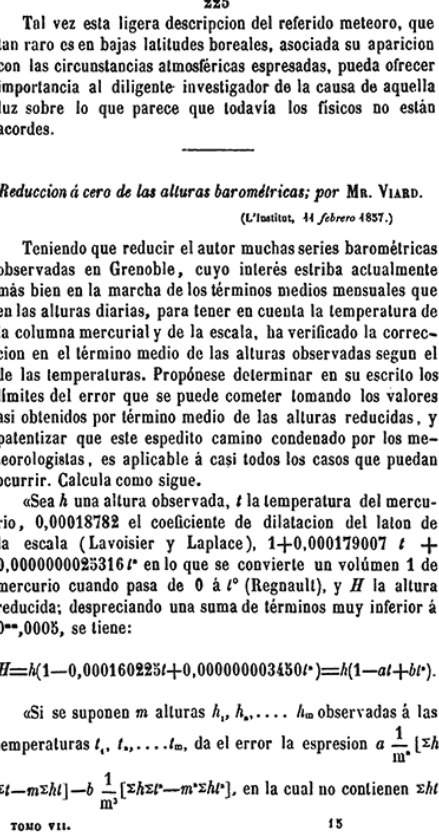

Figure 1. The original report in Spanish by Llanos (1857).

atmospheric optics or comet tails have been misinterpreted as auroral displays (e.g. Hayakawa et al., 2015, 2016a; Kawamura et al., 2016; Carrasco et al., 2017; Usoskin et al., 2017).

Its colour was described as "white" and less like lowlatitude auroras. However, due to the Purkinje effect, human eyes frequently perceive weak lights as apparently whitish, as they are insensitive to colour with weak brightness (Purkinje, 1825, p. 109; Minnaert, 1993, p. 133). Moreover, it was described as "a faint but weak white light on that horizon", and hence its brightness is considered rather faint and weak. Therefore, it is likely that this phenomenon is perceived as apparently whitish due to the Purkinje effect.

Atmospheric optics is dependent on the Moon for its light source (e.g. Minnaert, 1993). We computed the lunar phase on 27 October 1856, and obtained a value of 0.96 based on the method described by Kawamura et al. (2016) developed from Meeus (1988). This means that it was almost a new moon, and one can probably exclude the possibility that the light was associated with atmospheric optics from moonlight at night. Fogbows cannot explain this phenomenon either as they have a width of $25^{\circ}$ or greater, much smaller than normal rainbows, and they appear "nearly always ... when the dazzling beam of a car's headlights behind you penetrates the mist in front of you" (Minnaert, 1993, pp. 201-202). Llanos did not describe any such "dazzling beam behind" him.

Likewise, its descriptions of "width of $25^{\circ}$ or greater" and duration for "some 5 minutes" or " 10 minutes" show us that an upward discharge from the top of a thundercloud is also unlikely (e.g. Pasko et al., 2002), considering this glow was seen beyond the mountain ranges of Balanga and Zambales, about 60 and $140 \mathrm{~km}$ away from Manila respectively.

We also considered the possibility of a meteor shower. Within the October meteor showers listed in the catalogue of Kronk (2014, pp. 227-255), the Orionids are one of the candidates. However, Llanos reported that "At its base, the light was noticed to be more clear and perceptible", and it is unlikely that a meteor shower will decrease in brightness near the horizon. Moreover, the duration of 5 or $10 \mathrm{~min}$ is too short for a meteor shower. Likewise, it is also difficult to consider that this phenomenon might have been a comet tail as it lasted only $5 \mathrm{~min}$ in the NW and $10 \mathrm{~min}$ in the NE. Neither does Kronk (2003, pp. 245-246) report any comets in late 1856.

Mountain fire is also unlikely. While Llanos first suspected a fire in the mountains to be the cause, he had not gotten any reports of fire in the northern mountains of Manila, at least not until his publication. This phenomenon had a width of $25^{\circ}$ or greater and it would thus have to have been a large fire, which would have soon been reported to Manila if it were a fire in the mountains. Auroral displays are frequently mistaken for conflagrations when they are bright enough. In the Carrington event, a considerable number of observers in East Asia and North America misinterpreted the auroral displays as being conflagrations (Green et al., 2006; Hayakawa et al., 2016b). Similar reports are found during other large magnetic storms with bright auroral displays (Odenwald, 2007; Silverman, 2008; Vaquero et al., 2008; Ebihara et al., 2017; Hayakawa et al., 2017). 
It seems therefore that one has no strong reason to reject this as being one instance of "sporadic aurorae" which appear locally at relatively low MLAT, as reported in Silverman (2003). This case had a horizontal appearance of $\sim 25^{\circ}$ in width and $4^{\circ}$ in elevation. We would also note that it appeared in the north-westerly direction for $5 \mathrm{~min}$, and then in the north-easterly direction for $10 \mathrm{~min}$. Its base was brighter than the upper part, with "vertical undulation". These features also suggest its being interpreted as a kind of auroral display. Assuming that the altitude of the upper part of the aurora was $400 \mathrm{~km}$ (see Ebihara et al., 2017), we estimated that the aurora would have appeared at $19.5^{\circ}$ MLAT $\left(23.9^{\circ}\right.$ invariant latitude, ILAT, in the magnetic coordinates used to specify a magnetic field line in the space physics community). ILAT $\Lambda$ is constant along a field line, and is given by

$\Lambda=\cos ^{-1}(\sqrt{a / L})$

where $L$ is the distance in units of the Earth's radius between the centre of the Earth and the point where the magnetic field line crosses the equatorial plane (McIlwain, 1966). In contrast, MLAT $\lambda$ varies along a field line, and is given by

$\lambda=\cos ^{-1}(\sqrt{R / L})$,

where $R$ is the distance between the centre of the Earth and the specific point. At the surface of the Earth, $\Lambda$ is equal to $\lambda$.

\section{Contemporary solar and geomagnetic activities}

It is intriguing where this event is situated relative to solar and geomagnetic activities. It is known that the frequency of occurrence of magnetic storms is in relatively good agreement with the sunspot number (e.g. Vázquez et al., 2006; Willis et al., 2006), while recent statistical studies reveal that even the quieter Sun can on occasion also cause superstorms (e.g. Kilpua et al., 2015).

In terms of long-term solar activity, this event was mostly situated near the solar minimum in 1856 (e.g. Clette et al., 2014; Vaquero et al., 2016). The solar surface in October 1856 showed only a small number of sunspots (Carrington, 1863; Vaquero et al., 2016). Figure 2 shows the daily $a k$ value observed at Helsinki according to Nevanlinna (2004), indicating that the geomagnetic activity was also very low. Figure 3 shows the $H$ component of the geomagnetic field disturbances $(\Delta H)$ with a $1 \mathrm{~h}$ resolution. In the second half of the 19th century, a typical precision of a magnetometer was around $1^{\prime}$ (e.g. Batlloì, 2005) and may have caused apparently larger pseudo-random variations than those in the present day. On 27 October 1856, $\Delta \mathrm{H}$ at the Helsinki observatory (geographic latitude $60.2^{\circ}$ and geographic longitude $25.0^{\circ}$ ) exhibited a negative excursion, peaking at 15:00 UT, with an amplitude of $\sim 140 \mathrm{nT}$ as shown in Fig. 3a. The sporadic aurora occurred around

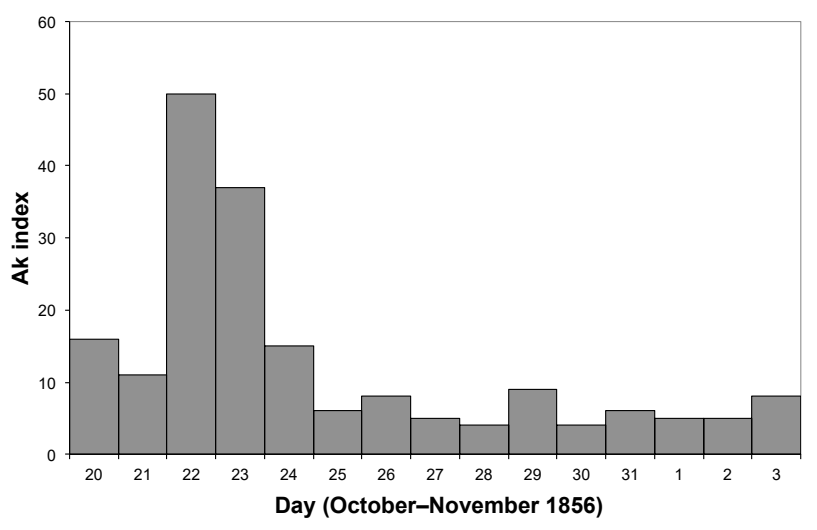

Figure 2. Daily $a k$ index (Nevanlinna, 1997) during the period 20 October-3 November 1856.

21:00-21:15 LT (12:56-13:11 UT) at Manila, which roughly corresponds to the descending phase of this negative excursion at Helsinki, by considering that the differences in time zones between Manila (N 14 $35^{\prime}$, E $\left.120^{\circ} 58^{\prime}\right)$, Helsinki observatory $\left(\mathrm{N} 60^{\circ} 10^{\prime}, \mathrm{E} 24^{\circ} 57^{\prime}\right)$, and Greenwich are roughly 7.07 and $8.06 \mathrm{~h}$ on the basis of local mean time (e.g. Nevanlinna, 2006, 2008). If this negative excursion is caused by the ring current, the secular variation is negligible, and the magnetic disturbance is independent of the magnetic local time, then the Dst would be calculated approximately as Dst $=\Delta H / \cos$ $\lambda$, where $\Delta H$ is the magnetic disturbance (Sugiura, 1964). Substituting $\Delta H$ of $\sim 140 \mathrm{nT}$ and $\lambda$ of $58.2^{\circ}$ (Helsinki observatory), we estimated Dst to be $\sim-266 \mathrm{nT}$. The recovery of the negative excursion takes place for only $1 \mathrm{~h}$, which is too short to attribute it to the decay of the storm-time ring current (Ebihara and Ejiri, 2003). The ionospheric current could also contribute to the variation of $\Delta H$. Figure $3 \mathrm{~b}$ shows $\Delta \mathrm{H}$ at the Lovö observatory $\left(59.3^{\circ} \mathrm{N}\right.$ and $\left.17.8^{\circ} \mathrm{E}\right)$ in the March 1989 storm. The Lovö observatory is close to Helsinki. To date, the March 1989 storm is the largest since 1957 in terms of the minimum Dst values $(-589 \mathrm{nT})$. The amplitude of $\Delta H$ exceeds $1000 \mathrm{nT}$, which is probably associated with the ionospheric current (in addition to other current systems such as the ring current), and is much larger than observed in Helsinki on 27 October 1856. Although the cause of the magnetic disturbance is uncertain, it can be said that the magnetic disturbance on 27 October 1856 was most likely low, at least at Helsinki, in comparison with the large storm in March 1989. Figure 3c shows $\Delta H$ at the Lovö observatory on 17-21 January 2002. The variation of $\Delta H$ on 19 January 2002 resembles that observed on 27 October 1856 in terms of the negative excursion and subsequent variation. The negative excursion starts at $\sim 12: 00 \mathrm{UT}$, and peaked at $\sim$ 16:00 UT on 19 January 2002. According to the OMNI solar wind data (King and Papitashvili, 2005), the negative excursion is associated with a southward turning of the interplanetary magnetic field (IMF) and a rapid increase in the 
(a)

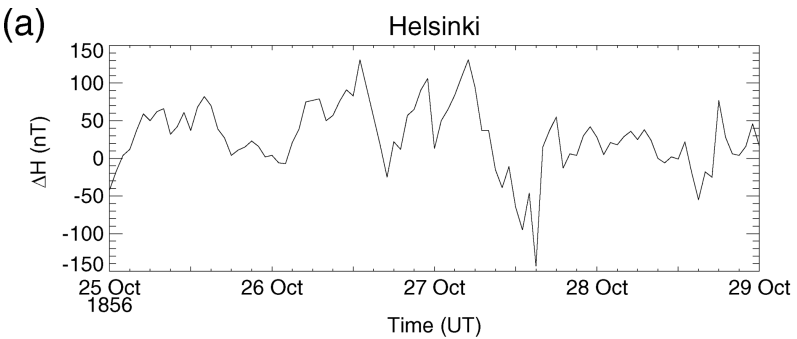

(b)

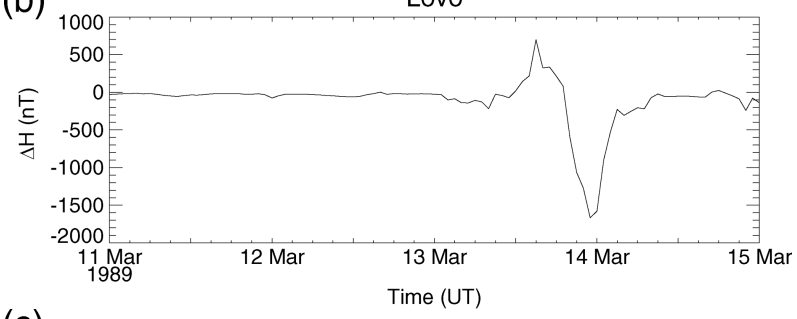

(c)

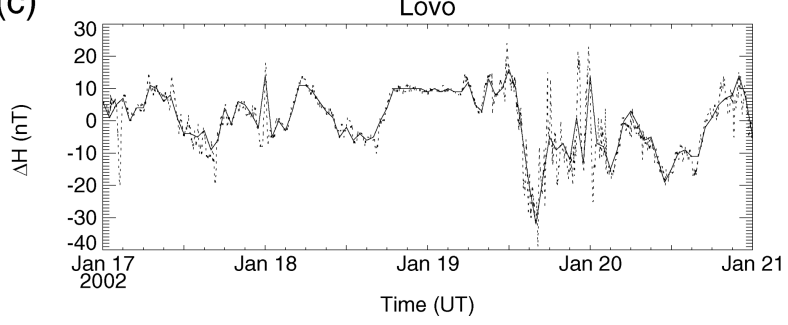

Figure 3. From (a) to (c), the $H$ component of the geomagnetic field disturbance at Helsinki in 1859, Lovö in 1989, and Lovö in 2002. The dotted line indicates the 1 min data.

solar wind dynamic pressure (data not shown). The sudden increase in the solar wind dynamic pressure resulted in the sudden increase in $\Delta H$, which is visible in the 1 min resolution data at Lovö (dotted line in Fig. 3c). The southward IMF continued until $\sim$ 15:00 UT, which could result in the intensification of the ring current and the negative variation of $\Delta H . \Delta H$ is highly fluctuating throughout this period, which is caused by fluctuations of the solar wind and IMF. The solar wind speed and density increased gradually, starting at $\sim$ 05:00 UT on 19 January 2002, and the strength of the IMF peaked at $\sim$ 09:00 UT on 19 January 2002. These characteristics may correspond to a corotating interaction region (CIR) (Denton et al., 2006). The Dst index did not reach $-30 \mathrm{nT}$ on 19-20 January 2002. The amplitude of the negative excursion $(\sim 140 \mathrm{nT})$ observed in 1856 is roughly 5 times larger than that observed in $2002(\sim-30 \mathrm{nT})$. This might indicate that the IMF Bz and/or solar wind velocity in 1856 was larger than those in 2002.

Therefore, we cannot find evidence of any strong geomagnetic disturbance on 27 October 1856 as in intense magnetic storms such as the superstorms in 1859 that brought auroral display down to a low MLAT (Kimball, 1960; Tsurutani et al., 2003; Cliver and Dietrich, 2013). One possible scenario is that a short-lasting magnetospheric disturbance occurred to cause the sporadic aurora. The disturbance is probably asso- ciated with a rapid enhancement of the magnetospheric electric field which transports magnetospheric electrons deeply earthwards (inwards). After being rapidly transported, the electrons were probably scattered by some processes on the field line at the $L$ value of 1.20 (23.9 ${ }^{\circ}$ ILAT). The scattered electrons could then have precipitated into the upper atmosphere, exciting oxygen atoms so as to cause the aurora. The disturbance should have been strong, at least at the $L$ value of 1.20 , but the duration should have been short (within at most $15 \mathrm{~min}$ ). If the duration of a strong disturbance (convection) is relatively long, hot ions also move inwards so as to intensify the plasma pressure (the ring current) that principally disturbs the geomagnetic field characterized by a negative excursion of the $H$ component of the magnetic field (Ebihara and Ejiri, 2003). The observation shows that the ring current was not strongly developed during this period. One of the possible causes of the short-lasting, large-amplitude disturbance is the interplanetary shock that reached the Earth. The compressional magnetospheric wave that was excited at the dayside magnetopause could propagate towards the Earth in the direction perpendicular to the magnetic field (e.g. Wilson and Sugiura, 1961). Shock-associated disturbances are observed in the magnetosphere at all magnetic local times at an $L$ value as low as $\sim 1.2$ (Shinbori et al., 2003, 2014). The transient compression of the magnetic field in the magnetosphere could result in the excitation of electromagnetic ion cyclotron (EMIC) waves (e.g. Immel et al., 2005) and chorus waves (e.g. Fu et al., 2012; Zhou et al., 2015). Interacting with the EMIC or chorus waves, the magnetospheric particles undergo pitch angle scattering, resulting in their precipitation into the upper atmosphere. According to observations, the wave intensifications and shock-associated auroras occur primarily on the dayside (e.g. Anderson and Hamilton, 1993; Zhang et al., 2004, 2008; Zhou et al., 2015). This seems to be inconsistent with the present aurora observation which was made at 21:00, local time. If the normal angle of the shock slants a lot, the impact of the interplanetary shock could be large enough in the late evening region (e.g. Selvakumaran et al., 2017) to excite EMIC and/or chorus waves at probably 21:00, local time.

Usually, the magnetic disturbance associated with an interplanetary shock lasts for just a few minutes to a few tens of minutes depending on solar wind dynamic pressure (Araki et al., 2004) and orientation angle of the shock front (Takeuchi et al., 2002). This short duration may explain why no significant disturbance was recorded in the daily $a k$ index as shown in Fig. 2, and in the hourly geomagnetic field data at Helsinki $\left(\mathrm{N} 60^{\circ} 10^{\prime}\right.$, E $\left.24^{\circ} 57^{\prime}\right)$ as shown in Fig. 3a. Since shock-associated magnetic disturbance is a global phenomenon (e.g. Nishida and Jacobs, 1962; Araki, 1994), the disturbance would have been detectable at Helsinki if the temporal resolution was high enough as shown in Fig. 3c. Due to its short duration, other observers may have missed it, instead seeing the clear sky at around "9 o'clock at night", Manila local time. This may explain why we have no auroral 
report on that same night at around $23.9^{\circ}$ ILAT, for example, from observers in East Asia (Willis et al., 2007; Kawamura et al., 2016).

\section{Conclusion}

In this short contribution, we have examined the record of an "aurora borealis" at Manila on 27 October 1856. According to our analysis of the text, we consider this record to indeed be likely one of an auroral display as was considered by the observer himself, Antonio Llanos. Reconstruction of contemporary MLAT showed that Manila was situated at $3.3^{\circ}$ MLAT, close to the geomagnetic equator. However, we could find no large sunspots or large geomagnetic storms associated with this auroral report. We did not find any contemporary auroral display reports in Willis et al. (2007) or Kawamura et al. (2016). This means that this auroral display was local at a low MLAT, and should be categorized as an instance of "sporadic auroras". In the analogy to the magnetic variation observed at Lovö in 2002, the sporadic aurora may be associated with a shock embedded in an interface of a corotating interaction region (CIR). The shock may result in transmission of an electromagnetic pulse propagating in the magnetosphere. In the course of the propagation, magnetospheric electrons could precipitate into the ionosphere, brightening the sporadic aurora. Further studies are needed to confirm this scenario in the future. As far as we know, this example is the first evidence of a sporadic aurora in Southeast Asia and near the geomagnetic equator. Together with known records of sporadic auroras from the United States (Silverman, 2003), East Asia (Willis et al., 2007), Spain (Vaquero et al., 2007), and Mexico (Vaquero et al., 2013), this record should provide a further resource with which to consider the physical nature of this phenomenon. Although this is rather an isolated phenomenon, further research into this phenomenon may merit studies of long-term variations of geomagnetic activity and the terrestrial magnetic field as well.

Data availability. The OMNI data were obtained from the GSFC/SPDF OMNI Web interface at https://omniweb.gsfc.nasa. gov (GSFC/SPDF, 2018).

Author contributions. $\mathrm{HH}$ originated the discussion and prepared the manuscript. JMV initiated the idea and collaboration, providing and translating the main historical source. YE constructed the possible scenario for this event. All authors contributed to the discussion and the writing of the final manuscript.

Competing interests. The authors declare that they have no conflict of interest.
Acknowledgements. The authors are indebted to Heikki Nevanlinna and Ari Viljanen, who provided the daily $a k$ index values, and the World Data Center for Geomagnetism, Kyoto, for providing the magnetic observation data. This research was also partially supported by the Economy and Infrastructure Board of the Junta of Extremadura through project IB16127 and grant GR15137 (co-financed by the European Regional Development Fund), the Ministerio de Economía y Competitividad of the Spanish Government (AYA2014-57556-P and CGL2017-87917-P), a grant-inaid from the Ministry of Education, Culture, Sports, Science and Technology of Japan, grant numbers JP15H05816 (PI: Shigeo Yoden), JP15H03732 (PI: Yusuke Ebihara), JP16H03955 (PI: Kazunari Shibata), JP 18H01254 (PI: Hiroaki Isobe), and JP15H05815 (PI: Yoshizumi Miyoshi), a grant-in-aid for JSPS Research Fellow JP17J06954 (PI: Hisashi Hayakawa), and the Exploratory and Mission Research Projects of the Research Institute for Sustainable Humanosphere (PI: Hiroaki Isobe). The authors gratefully thank Sam M. Silverman for attracting our attention to the sporadic aurora and Tiera Laitinen and another anonymous referee for their helpful and constructive comments on our paper.

The topical editor, Ana G. Elias, thanks Tiera Laitinen and one anonymous referee for help in evaluating this paper.

\section{References}

Abbe, C.: An aurora in South Carolina and Kentucky, Mon. Weather Rev., 23, 297-298, 1895.

Allen, J., Frank, L., Sauer, H., and Reiff, P.: Effects of the March 1989 solar activity, EOS, 70, 1486-1488, https://doi.org/10.1029/89EO00409, 1989.

Anderson, B. J. and Hamilton, D. C.: Electromagnetic ion cyclotron waves stimulated by modest magnetospheric compressions, J. Geophys. Res., 98, 11369-11382, https://doi.org/10.1029/93JA00605, 1993.

Araki, T.: A Physical Model of the Geomagnetic Sudden Commencement, in: Solar Wind Sources of Magnetospheric UltraLow-Frequency Waves, edited by: Engebretson, M. J., Takahashi, K. and Scholer, M., American Geophysical Union, Washington, DC, USA, https://doi.org/10.1029/GM081p0183, 1994.

Araki, T., Takeuchi, T., and Araki, Y.: Rise time of geomagnetic sudden commencements - Statistical analysis of ground geomagnetic data, Earth Planet. Space, 56, 289-293, https://doi.org/10.1186/BF03353411, 2004.

Batlloì, J.: Cataòlogo inventario de magnetoìmetros españoles, Centro Nacional de Informacion Geograìfica, Madrid, Spain, 2005.

Botley, C. M.: Sporadic aurora, Planet. Space Sci., 11, 723-724, 1963.

Carrasco, V. M. S., Trigo, R., and Vaquero, J. M.: Unusual rainbows as auroral candidates: Another point of view, P. Astron. Soc. Jpn., 69, L1, https://doi.org/10.1093/pasj/psw127, 2017.

Carrington, R. C.: Observations of the spots on the sun from November 9, 1853, to March 24, 1861, made at Redhill, William \& Norgate, London, UK, 1863.

Clette, F., Svalgaard, L., Vaquero, J. M., and Cliver, E. W.: Revisiting the Sunspot Number. A 400-Year Perspective on the Solar Cycle, Space Sci. Rev., 186, 35-103, https://doi.org/10.1007/s11214-014-0074-2, 2014. 
Cliver, E. W. and Dietrich, W. F.: The 1859 space weather event revisited: limits of extreme activity, J. Space Weather Spac., 3, A31, https://doi.org/10.1051/swsc/2013053, 2013.

Cliver, E. W. and Svalgaard, L.: The 1859 Solar-Terrestrial Disturbance And the Current Limits of Extreme Space Weather Activity, Sol. Phys., 224, 407-422, https://doi.org/10.1007/s11207005-4980-z, 2004.

Denton, M. H., Borovsky, J. E., Skoug, R. M., Thomsen, M. F., Lavraud, B., Henderson, M. G., McPherron, R. L., Zhang, J. C., and Liemohn, M. W.: Geomagnetic storms driven by ICMEand CIR-dominated solar wind, J. Geophys. Res., 111, A07S07, https://doi.org/10.1029/2005JA011436, 2006.

Ebihara, Y. and Ejiri, M.: Numerical simulation of the ring current, Space Sci. Rev., 105, 377-452, 2003.

Ebihara, Y., Hayakawa, H., Iwahashi, K., Tamazawa, H., Kawamura, A. D., and Isobe, H.: Possible cause of extremely bright aurora witnessed in East Asia on 17 September 1770, Space Weather, 15, 1373-1382, https://doi.org/10.1002/2017SW001693, 2017.

Farrona, A. M., Gallego, M. C., Vaquero, J. M., and DomínguezCastro, F.: Spanish Eyewitness Accounts of the Great Space Weather Event of 1859, Acta Geod. Geophys. Hu., 46, 370-377, https://doi.org/10.1556/AGeod.46.2011.3.7, 2011.

Fu, H. S., Cao, J. B., Mozer, F. S., Lu, H. Y., and Yang, B.: Chorus intensification in response to interplanetary shock, J. Geophys. Res., 117, A01203, https://doi.org/10.1029/2011JA016913, 2012.

Gonzalez, W. D., Joselyn, J. A., Kamide, Y., Kroehl, H. W., Rostoker, G., Tsurutani, B. T., and Vasyliunas, V. M.: What is a geomagnetic storm?, J. Geophys. Res., 99, 5771-5792, https://doi.org/10.1029/93JA02867, 1994.

Green, J. and Boardsen, S.: Duration and extent of the great auroral storm of 1859, Adv. Space Res., 38, 130-135, https://doi.org/10.1016/j.asr.2005.08.054, 2006.

Green, J. L., Boardsen, S., Odenwald, S., Humble, J., and Pazamickas, K. A. Eyewitness reports of the great auroral storm of 1859, Adv. Space Res., 38, 145-154, 2006.

GSFC/SPDF: OMNI data, available at: https://omniweb.gsfc.nasa. gov, last access: 20 August 2018.

Hayakawa, H., Tamazawa, H., Kawamura, A. D., and Isobe, H.: Records of sunspot and aurora during CE 960-1279 in the Chinese chronicle of the Sòng dynasty, Earth. Planet. Space, 67, 82, https://doi.org/10.1186/s40623-015-0250-y, 2015.

Hayakawa, H., Isobe, H., Kawamura, A. D., Tamazawa, H., Miyahara, H., and Kataoka, R.: Unusual rainbow and white rainbow: A new auroral candidate in oriental historical sources, Publ. Astron. Soc. Jpn., 68, 33, https://doi.org/10.1093/pasj/psw032, 2016a.

Hayakawa, H., Iwahashi, K., Tamazawa, H., Isobe, H., Kataoka, R., Ebihara, Y., Miyahara, H., Kawamura, A. D., and Shibata, K.: East Asian observations of low latitude aurora during the Carrington magnetic storm, Publ. Astron. Soc. Jpn., 68, 99, https://doi.org/10.1093/pasj/psw097, 2016b.

Hayakawa, H., Iwahashi, K., Ebihara, Y., Tamazawa, H., Shibata, K., Knipp, D. J., Kawamura, A. D., Hattori, K., Mase, K., Nakanishi, I., and Isobe, H.: Long-lasting Extreme Magnetic Storm Activities in 1770 Found in Historical Documents, Astrophys. J. Lett., 850, L31, https://doi.org/10.3847/2041-8213/aa9661, 2017.
Hayakawa, H., Ebihara, Y., Vaquero, J. M., Hattori, K., Carrasco, V. M. S., Gallego, M. C., Hayakawa, S., Watanabe, Y., Iwahashi, K., Tamazawa, H., Kawamura, A. D., and Isobe, H.: A great space weather event in February 1730, Astron. Astrophys., https://doi.org/10.1051/0004-6361/201832735, 2018 a.

Hayakawa, H., Ebihara, Y., Willis, D. M., Hattori, K., Giunta, A. S., Wild, M. N., Hayakawa, S., Toriumi, S., Mitsuma, Y., Macdonald, L. T., Shibata, K., and Silverman, S. M.: The Great Space Weather Event during February 1872 Recorded in East Asia, Astrophys. J., 862, 15, https://doi.org/10.3847/1538-4357/aaca40, 2018b.

Immel, T. J., Mende, S. B., Frey, H. U., Patel, J., Bonnel, H. W., Engebretson, M. J., and Fuselier, S. A.: ULF waves associated with enhanced sub-auroral proton precipitation, Geophys. Monogr. Ser., 159, 71, 2005.

Jackson, A., Jonkers, A. R. T., and Walker, M.: Four centuries of geomagnetic secular variation from historical records, Philos. T. R. Soc. A, 358, 957, 2000.

Kawamura, A. D., Hayakawa, H., Tamazawa, H., Miyahara, H., and Isobe, H.: Aurora candidates from the chronicle of Qíng dynasty in several degrees of relevance, Publ. Astron. Soc. Jpn,, 68, 79, https://doi.org/10.1093/pasj/psw074, 2016.

Kilpua, E. K. J., Olspert, N., Grigorievskiy, A., Käpylä, M. J., Tanskanen, E. I., Miyahara, H., Kataoka, R., Pelt, J., and Liu, Y. D.: Statistical Study of Strong and Extreme Geomagnetic Disturbances and Solar Cycle Characteristics, Astrophys. J., 806, 272, https://doi.org/10.1088/0004-637X/806/2/272, 2015.

Kimball, D. S.: A study of the aurora of 1859. Scientific Report No. 6, University of Alaska, No. 6, 1960.

King, J. H. and Papitashvili, N. E.: Solar wind spatial scales in and comparisons of hourly Wind and ACE plasma and magnetic field data, J. Geophys. Res., 110, A02209, 10.1029/2004JA010649, 2005.

Kronk, G. W.: Cometography: A Catalog of Comets, III, Cambridge University Press, Cambridge, UK, 2003.

Kronk, G. W.: Meteor Showers, an Annotated Catalog, Springer, New York, USA, 2014.

Lakhina, G. S. and Tsurutani, B. T.: Geomagnetic storms: historical perspective to modern view, Geosci. Lett., 3, 5, https://doi.org/10.1186/s40562-016-0037-4, 2016.

Llanos, A.: Observación de una aurora boreal en Manila, Revista de los Progresos de las Ciencias Exactas, Físicas y Naturales, 7, 223-225, 1857.

McIlwain, C. E.: Magnetic coordinates, Space Sci. Rev., 5, 585598, 1966.

Meeus, J.: Astronomical algorithms, 2nd ed., Richmond, WillmannBell, VA, USA, 1998.

Minnaert, M. G. J.: Light and Color in the Outdoors, Springer, New York, USA, 1993.

Nevanlinna, H.: Gauss' H-Variometer at the Helsinki Magnetic Observatory (1844-1912), J. Geomagn. Geoelectr., 49, 1209-1216, https://doi.org/10.5636/jgg.49.1209, 1997.

Nevanlinna, H.: Results of the Helsinki magnetic observatory 1844-1912, Ann. Geophys., 22, 1691-1704, https://doi.org/10.5194/angeo-22-1691-2004, 2004.

Nevanlinna, H.: A study on the great geomagnetic storm of 1859: Comparisons with other storms in the 19th century, Adv. Space Res., 38, 180-187, 2006. 
Nevanlinna, H. and Kataja, E.: An extension of the geomagnetic activity index series aa for two solar cycles (1844-1868), Geophys. Res. Lett., 20, 2703-2706, 1993.

Nishida, A. and Jacobs, J. A.: World-wide changes in the geomagnetic field, J. Geophys. Res., 67, 525-540, https://doi.org/10.1029/JZ067i002p00525, 1962.

Odenwald, S.: Newspaper reporting of space weather: End of a golden age, Adv. Space Res., 5, S11005, https://doi.org/10.1029/2007SW000344, 2007.

Odenwald, S.: Solar Storms: 2000 years of human calamity! Createspace Independent Publishing Platform, San Bernardino, CA, USA, 2015.

Pasko, V. P., Stanley, M. A., Mathews, J. D., Inan, U. S., and Wood, T. G.: Electrical discharge from a thundercloud top to the lower ionosphere, Nature, 416, 152-154, https://doi.org/10.1038/416152a, 2002.

Purkinje, J. E.: Neue Beiträge zur Kenntniss des Sehens in Subjectiver Hinsicht (Berlin: Reimer), 1825.

Ribeiro, P., Vaquero, J. M., and Trigo, R.: Geomagnetic records of Carrington's storm from Guatemala, J. Atmos. Sol.-Terr. Phy., 73, 308-315, 2011.

Selvakumaran, R., Veenadhari, B., Ebihara, Y., Kumar, S., and Prasad, D. S.: The role of interplanetary shock orientation on SC/SI rise time and geoeffectiveness, Adv. Space Res., 59, 14251434, https://doi.org/10.1016/j.asr.2016.12.010, 2017.

Shinbori, A., Ono, T., Izima, M., Kumamoto, A., and Oya, H.: Sudden commencements related plasma waves observed by the Akebono satellite in the polar region and inside the plasmasphere region, J. Geophys. Res., 108, 1457, https://doi.org/10.1029/2003JA009964, 2003.

Shinbori, A., Ono, T., Iizima, M., and Kumamoto, A.: SC related electric and magnetic field phenomena observed by the Akebono satellite inside the plasmasphere, Earth Planet. Space., 56, 269, https://doi.org/10.1186/BF03353409, 2014.

Shiokawa, K., Meng, C.-I., Reeves, G. D., Rich, F. J., and Yumoto, K.: A multievent study of broadband electrons observed by the DMSP satellites and their relation to red aurora observed at midlatitude stations, J. Geophys. Res., 102, 14237-14253, https://doi.org/10.1029/97JA00741, 1998.

Shiokawa, K., Ogawa, T., and Kamide, Y.: Low-latitude auroras observed in Japan: 1999-2004, J. Geophys. Res., 110, A05202, https://doi.org/10.1029/2004JA010706, 2005.

Silverman, S. M.: Low latitude auroras: the storm of 25 September 1909, J. Atmos. Terr. Phys., 57, 673-685, 1995.

Silverman, S. M.: Sporadic auroras, J. Geophys. Res., 108, A4, https://doi.org/10.1029/2002JA009335, 2003.

Silverman, S. M.: Comparison of the aurora of September 1/2, 1859 with other great auroras, Adv. Space Res., 38, 136-144, 2006.

Silverman, S. M.: Low-latitude auroras: The great aurora of 4 February 1872, J. Atmos. Sol.-Terr. Phy., 70, 1301-1308, 2008.

Silverman, S. M. and Cliver, E. W.: Low-latitude auroras: the magnetic storm of 14-15 May 1921, J. Atmos. Sol.-Terr. Phy., 63, 523-535, 2001.

Sugiura, M.: Hourly values of equatorial Dst for the IGY, Ann. Int. Geophys. Year, Pergamon Press, Oxford, UK, 35, 1964.

Takeuchi, T., Russell, C. T., and Araki, T.: Effect of the orientation of interplanetary shock on the geomagnetic sudden commencement, J. Geophys. Res., 107, 1423, https://doi.org/10.1029/2002JA009597, 2002.
Thébault, E., Finlay, C. C., Beggan, C. D., et al.: International Geomagnetic Reference Field: the 12th generation, Earth Planet Space, 67, 1, https://doi.org/10.1186/s40623-015-0228-9, 2015.

Tsurutani, B. T., Gonzales, W. D., Lakhina, G. S., and Alex, S.: The extreme magnetic storm of 1-2 September 1859, J. Geophys. Res., 108, 1268, https://doi.org/10.1029/2002JA009504, 2003.

Usoskin, I. G., Kovaltsov, G. A., Mishina, L. N., Sokoloff, D. D., and Vaquero, J.: An Optical Atmospheric Phenomenon Observed in 1670 over the City of Astrakhan Was Not a Mid-Latitude Aurora, Sol. Phys., 292, 15, https://doi.org/10.1007/s11207-0161035-6, 2017.

Vaquero, J. M., Gallego, M. C., and García, J. A.: Early meteorological records of Manila: El Niño episode of 1864, Atmósfera, 18, 245-258, 2005.

Vaquero, J. M., Trigo, R., and Gallego, M. C.: Sporadic Aurora in Spain, Earth Planet. Space, 59, e49-e51, 2007.

Vaquero, J. M., Valente, M. A., Trigo, R. M., and Gallego, M. C.: The 1870 Space Weather Event: Geomagnetic and Auroral Records, J. Geophys. Res., 113, A08230, https://doi.org/10.1029/2007JA012943, 2008.

Vaquero, J. M., Gallego, M. C., and Domínguez-Castro, F.: A possible case of Sporadic Aurora in 1843 from Mexico, Geofís. Int., 52, 87-92, 2013.

Vaquero, J. M., Svalgaard, L., Carrasco, V. M. S., Clette, F., Lefèvre, L., Gallego, M. C., Arlt, R., Aparicio, A. J. P., Richard, J.-G., and Howe, R.: A Revised Collection of Sunspot Group Numbers, Sol. Phys., 291, 3061-3074, https://doi.org/10.1007/s11207016-0982-2, 2016.

Vázquez, M., Vaquero, J. M., and Curto, J. J.: On the Connection Between Solar Activity and Low-Latitude Aurorae in the Period 1715-1860, Sol. Phys., 238, 405-420, https://doi.org/10.1007/s11207-006-0194-2, 2006.

Willis, D. M., Henwood, R., and Stephenson, F. R.: The presence of large sunspots near the central solar meridian at the times of modern Japanese auroral observations, Ann. Geophys., 24, 2743-2758, 2006.

Willis, D. M., Stephenson, F. R., and Huiping Fang: Sporadic aurorae observed in East Asia, Ann. Geophys., 25, 417-436, https://doi.org/10.5194/angeo-25-417-2007, 2007.

Wilson, C. R. and Sugiura, M.: Hydromagnetic interpretation of sudden commencements of magnetic storms, J. Geophys. Res., 66, 12, 4097-4111, https://doi.org/10.1029/JZ066i012p04097, 1961.

Yokoyama, N., Kamide, Y., and Miyaoka, H.: The size of the auroral belt during magnetic storms, Ann. Geophys., 16, 566-573, https://doi.org/10.1007/s00585-998-0566-z, 1998.

Zhang, Y., Paxton, L. J., Meng, C.-I., Morrison, D., Wolven, B., Kil, H., and Christensen, A. B.: Double dayside detached auroras: TIMED/GUVI observations, Geophys. Res. Lett., 31, L10801, https://doi.org/10.1029/2003GL018949, 2004.

Zhang, Y., Paxton, L. J., and Zheng, Y.: Interplanetary shock induced ring current auroras, J. Geophys. Res., 113, A01212, https://doi.org/10.1029/2007JA012554, 2008.

Zhou, C., Li, W., Thorne, R. M., Bortnik, J., Ma, Q., An, X., Zhang, X., Angelopoulos, V., Ni, B., Gu, X., Fu, S., and Zhao, Z.: Excitation of dayside chorus waves due to magnetic field line compression in response to interplanetary shocks, J. Geophys. Res.Space, 120, 8327-8338, https://doi.org/10.1002/2015JA021530, 2015. 